\title{
A Note on Positive Periodic Solutions of the Superlinear Heat Equation with Inhomogeneity
}

\author{
Shanming Ji, Jingxue Yin and Jian Deng \\ School of Mathematical Sciences, South China Normal University, Guangzhou 510631, China \\ Email: dengjian@scnu.edu.cn
}

\begin{abstract}
This paper deals with the existence of positive periodic solutions of the superlinear heat equation with inhomogeneity. We first prove the existence of positive periodic solutions to the equation with an indispensable nontrivial and globally small inhomogeneity; and then the non-existence for a locally large inhomogeneity with possibly small support is obtained.
\end{abstract}

Keywords: Positive periodic solutions, locally large inhomogeneity, globally small inhomogeneity.

\section{Introduction}

Let $\Omega$ be a bounded domain in $\mathbb{R}^{N}$ with smooth boundary $\partial \Omega$ and $f(x, t), m(x, t)$ be two positive functions which are $T$-periodic with respect to the time $t$. We are concerned with the existence of positive periodic solutions for the following inhomogeneous superlinear heat equation

$$
\left\{\begin{array}{l}
\frac{\partial u}{\partial t}-\Delta u=m(x, t) u^{q}+f(x, t), \quad(x, t) \in \Omega \times \mathbb{R}^{+} \\
u(x, t)=0, \quad(x, t) \in \partial \Omega \times \mathbb{R}^{+} \\
u(x, t)=u(x, t+T), \quad(x, t) \in \Omega \times \mathbb{R}^{+},
\end{array}\right.
$$

where $q>1$.

The periodic problem (1) with $f \equiv 0$ has been intensively studied by many authors since the last century. It is known that there exists at least one positive periodic solution if $1<q<(N+2) /(N-2)$ $(q>1$ for $N=1,2)$ and no positive periodic solution provided that $q \geq(N+2) /(N-2)$ with $N>2$ and $\Omega$ is strictly star-shaped, see $[1,2,6,7,8]$ and references therein. As for the inhomogeneous case $f \not \equiv 0$, it was Ôtani [5, J. Diff. Equations, 1984] who proved that there exists a periodic solution for (1) if $f$ is sufficiently small with $1<q<(N+2) /(N-2)$. Further, Hirano and Mizoguchi [3, Proc. Amer. Math. Soc., 1995] showed that there exist a stable and an unstable positive periodic solutions for the problem (1) under similar conditions.

The purpose of the present note is to give a complete characterization for the existence and non-existence of nontrivial periodic solutions for all the possible value of the exponent $q>1$. As a supplementation, we obtain the existence of the positive periodic solution for the indispensable nontrivial and globally small inhomogeneity $f(x, t)$ with $q \geq(N+2) /(N-2)$, and we also find the non-existence result for the locally large inhomogeneity $f(x, t)$ with possibly small support and $q>1$.

For simplicity, we put $Q_{T}=\Omega \times(0, T)$ and let $B_{r}\left(x_{0}\right)$ be the ball with radius $r$ centered at $x_{0}$. Throughout this paper we assume that $q>1$ and

$$
\underline{m} \leq m(x, t) \leq \bar{m}, \quad f(x, t) \geq 0, \quad f(x, t) \not \equiv 0, \quad(x, t) \in Q_{T}
$$

for some positive constants $\bar{m} \geq \underline{m}>0$.

We state our main results here.

Theorem 1.1 For any $x_{0} \in \Omega$ and $r>0$, the problem (1) admits no positive periodic solution provided that $\inf _{\left(B_{r}\left(x_{0}\right) \cap \Omega\right) \times(0, T)} f(x, t)$ is sufficiently large; while, the problem (1) admits at least one positive periodic solution provided that $\sup _{Q_{T}} f(x, t)$ is sufficiently small. 


\section{Proof of the main results}

Before proving the non-existence result, we present a lower bound estimate based on the comparison principle with weak lower solutions.

Lemma 2.1 Suppose that $u, v \in W_{2}^{1,1}\left(Q_{T}\right)$ such that

$$
\frac{\partial u}{\partial t}-\Delta u \geq \frac{\partial v}{\partial t}-\Delta v, \quad(x, t) \in Q_{T}
$$

in the sense of distributions, and $u, v$ satisfy $u(x, T)=u(x, 0), v(x, T)=v(x, 0)$ for $x \in \Omega, u(x, t) \geq v(x, t)$ for $x \in \partial \Omega, t \in(0, T)$. Then $u(x, t) \geq v(x, t)$ for $(x, t) \in Q_{T}$.

Proof. Let $z=v-u$ and $\varphi=(v-u)_{+}$. Then $\varphi, z \in W_{2}^{1,1}, \varphi$ and $z$ are $T$-periodic with respect to $t$, $\varphi(x, t)=0$ for $x \in \partial \Omega$, and

$$
z_{t}-\Delta z \leq 0, \quad(x, t) \in Q_{T}
$$

in the sense of distributions. Taking $\varphi$ as the test function, we see that

$$
\iint_{Q_{T}}\left(\varphi \varphi_{t}+|\nabla \varphi|^{2}\right) \mathrm{d} x \mathrm{~d} t \leq 0
$$

Combining with the periodicity and the Poincaré inequality, we have $\varphi=0$ a.e. $Q_{T}$.

Lemma 2.2 Assume that $u$ is the solution of the following problem

$$
\begin{cases}-\Delta u=g, & x \in \Omega, \\ u(x)=0, & x \in \partial \Omega,\end{cases}
$$

where $g(x) \geq 0$ for $x \in \Omega$, and $g(x) \geq K>0$ for $x \in B_{r}\left(x_{0}\right) \subset \Omega$. Then $u(x) \geq 0$ for $x \in \Omega$, and $u(x) \geq \frac{K}{4 N} r^{2}$ for $x \in B_{r / \sqrt{2}}\left(x_{0}\right)$.

Proof. Let

$$
v(x)=\frac{K}{2 N}\left(r^{2}-\left|x-x_{0}\right|^{2}\right)_{+}
$$

Then $v \in H_{0}^{1}(\Omega)$ and

$$
-\Delta v \leq K \chi_{B_{r}\left(x_{0}\right)} \leq g(x), \quad x \in \Omega
$$

in the sense of distributions, where $\chi_{B_{r}\left(x_{0}\right)}$ is the characteristic function of the ball $B_{r}\left(x_{0}\right)$. Lemma 2.1 implies that

$$
u(x) \geq v(x) \geq \frac{K}{4 N} r^{2} \chi_{B_{r / \sqrt{2}}\left(x_{0}\right)} .
$$

The proof is completed.

Lemma 2.3 For any $q>1, x_{0} \in \Omega$ and $r>0$, there exists a positive constant $K=K\left(q, r, x_{0}\right)$, such that if

$$
\inf _{(x, t) \in\left(B_{r}\left(x_{0}\right) \cap \Omega\right) \times(0, T)} f(x, t) \geq K,
$$

then the problem (1) admits no positive periodic solution.

Proof. Assume that $u(x, t)$ is a positive periodic solution of the problem (1). Since $x_{0} \in \Omega$, we can choose $x_{0}^{\prime} \in \Omega$ and $r^{\prime}>0$ such that $B_{r^{\prime}}\left(x_{0}^{\prime}\right) \subset \Omega \cap B_{r}\left(x_{0}\right)$. Without loose of generality, we assume that $B_{r}\left(x_{0}\right) \subset \Omega$. Let $u_{1}$ be the solution of the following problem

$$
\begin{cases}-\Delta u_{1}=K \chi_{B_{r}\left(x_{0}\right)}, & x \in \Omega, \\ u_{1}(x)=0, & x \in \partial \Omega,\end{cases}
$$


where $\chi_{B_{r}\left(x_{0}\right)}$ is the characteristic function of the ball $B_{r}\left(x_{0}\right)$. Then $u_{1} \geq 0$ in $\Omega$ and

$$
u_{1}(x) \geq \frac{K}{4 N} r^{2}, \quad x \in B_{\frac{r}{\sqrt{2}}}\left(x_{0}\right)
$$

according to Lemma 2.2. Furthermore, we see that

$$
u(x, t) \geq u_{1}(x), \quad(x, t) \in Q_{T}
$$

from Lemma 2.1. Consider the following problem

$$
\begin{cases}-\Delta u_{2}=\underline{m} u_{1}^{q}, & x \in \Omega, \\ u_{2}(x)=0, & x \in \partial \Omega .\end{cases}
$$

We fix a constant $d>0$ such that

$$
\left(\frac{d}{2}\right)^{q} \geq d, \quad d^{2} \geq 2^{N / 2+3} .
$$

This can be done by choosing $d$ sufficiently large since $q>1$. And then we choose $K$ sufficiently large such that

$$
\underline{m}\left(\frac{r^{2}}{4 N}\right)^{q} K^{q-1} \geq d
$$

since $q>1$. Now, we have

$$
\underline{m} u_{1}^{q} \geq \underline{m}\left(\frac{K}{4 N} r^{2}\right)^{q} \geq d K, \quad x \in B_{\frac{r}{\sqrt{2}}}\left(x_{0}\right) .
$$

Lemma 2.2 implies that $u_{2} \geq 0$ in $\Omega$ and

$$
u_{2}(x) \geq \frac{d K}{4 N}\left(\frac{r}{\sqrt{2}}\right)^{2}, \quad x \in B_{\frac{r}{2}}\left(x_{0}\right) .
$$

Similarly, we see that

$$
u(x, t) \geq u_{2}(x), \quad(x, t) \in Q_{T}
$$

from Lemma 2.1. In general, for $n \geq 2$, we consider the following problem

$$
\begin{cases}-\Delta u_{n+1}=\underline{m} u_{n}^{q}, & x \in \Omega, \\ u_{n+1}(x)=0, & x \in \partial \Omega .\end{cases}
$$

We prove by induction that

$$
u_{n}(x) \geq \frac{d^{n-1} K}{4 N} \frac{r^{2}}{2^{n-1}}, \quad x \in B_{\frac{r}{2^{n / 2}}}\left(x_{0}\right),
$$

and

$$
u(x, t) \geq u_{n}(x), \quad(x, t) \in Q_{T}
$$

for $n \in \mathbb{N}$. The cases $n=1,2$ have already been proved above. Suppose that for some $i \in \mathbb{N}$ the assertion is true. We can verify that

$$
\underline{m} u_{i}^{q} \geq \underline{m}\left(\frac{d^{i-1} K}{4 N} \frac{r^{2}}{2^{i-1}}\right)^{q} \geq d K\left(\frac{d}{2}\right)^{q(i-1)} \geq d^{i} K, \quad x \in B_{\frac{r}{2^{n / 2}}}\left(x_{0}\right),
$$

according to (2) and (3). Applying Lemma 2.2, we see that

$$
u_{i+1}(x) \geq \frac{d^{i} K}{4 N}\left(\frac{r}{2^{n / 2}}\right)^{2}, \quad x \in B \frac{r}{2^{(n+1) / 2}}\left(x_{0}\right) .
$$


The induction is completed by using the comparison principle Lemma 2.1. Thus,

$$
\begin{aligned}
\|u\|_{L^{2}\left(Q_{T}\right)}^{2} & \geq T\left\|u_{n+1}\right\|_{L^{2}(\Omega)}^{2} \\
& \geq \omega_{N} T\left(\frac{d^{n} K}{4 N} \frac{r^{2}}{2^{n}}\right)^{2}\left(\frac{r}{2^{(n+1) / 2}}\right)^{N} \\
& =\omega_{N} T\left(\frac{K r^{2}}{4 N}\right)^{2}\left(\frac{r}{\sqrt{2}}\right)^{N}\left(\frac{d^{2}}{2^{N / 2+2}}\right)^{n} \\
& \geq \omega_{N} T\left(\frac{K r^{2}}{4 N}\right)^{2}\left(\frac{r}{\sqrt{2}}\right)^{N} \cdot 2^{n}
\end{aligned}
$$

from $(2)$, where $\omega_{N}$ is the measure of $B_{1}(0)$. Hence we have obtained a contradiction to the fact that $u \in L^{2}\left(Q_{T}\right)$.

Finally, we employ the monotone iteration technique to show the existence results.

Lemma 2.4 For any $q>1$ and $s>\frac{N+2}{2}$, there exists a positive constant $\varepsilon=\varepsilon(q, s)$, such that if $\|f\|_{L^{s}\left(Q_{T}\right)} \leq \varepsilon$ then the problem (1) admits at least one positive periodic solution.

Proof. Let $u_{1}(x, t)$ be the periodic solution of the following problem

$$
\begin{cases}\frac{\partial u_{1}}{\partial t}-\Delta u_{1}=f(x, t), & (x, t) \in Q_{T}, \\ u_{1}(x, t)=0, & (x, t) \in \partial \Omega \times(0, T), \\ u_{1}(x, 0)=u_{1}(x, T), & x \in \Omega .\end{cases}
$$

The existence and uniqueness is trivial. Since $f \geq 0$ and $f \not \equiv 0$, we see that $u_{1}$ is a positive periodic function. For $n \geq 1$, let $u_{n+1}$ be the positive periodic solution of the following problem

$$
\begin{cases}\frac{\partial u_{n+1}}{\partial t}-\Delta u_{n+1}=m(x, t) u_{n}^{q}+f(x, t), & (x, t) \in Q_{T}, \\ u_{n+1}(x, t)=0, & (x, t) \in \partial \Omega \times(0, T), \\ u_{n+1}(x, 0)=u_{n+1}(x, T), & x \in \Omega .\end{cases}
$$

Since $u_{1}>0$, we see that $u_{2} \geq u_{1}$. By the induction, we have $u_{n+1} \geq u_{n} \geq \cdots \geq u_{1}>0$.

Next we show that the sequence $\left\{u_{n}\right\}$ converges provided that $\|f\|_{L^{s}\left(Q_{T}\right)}$ is sufficiently small. The classical result in [4] implies that

$$
\left\|u_{1}\right\|_{L^{\infty}\left(Q_{T}\right)} \leq C_{0}\|f\|_{L^{s}\left(Q_{T}\right)}
$$

where $C_{0}$ is a positive constant. We choose $\varepsilon>0$ sufficiently small such that

$$
\bar{m}\left|Q_{T}\right|^{\frac{1}{s}}\left(2 C_{0}\right)^{q} \varepsilon^{q-1} \leq 1
$$

since $q>1$. Now, for $\|f\|_{L^{s}\left(Q_{T}\right)} \leq \varepsilon$, we prove by induction that

$$
\left\|u_{n}\right\|_{L^{\infty}\left(Q_{T}\right)} \leq 2 C_{0}\|f\|_{L^{s}\left(Q_{T}\right)}, \quad n \in \mathbb{N} .
$$

The case $n=1$ has been proved above. Assume that for some $i \in \mathbb{N}$ the estimate (4) is true. Therefore, we have

$$
\begin{aligned}
\left\|u_{i+1}\right\|_{L^{\infty}\left(Q_{T}\right)} & \leq C_{0}\left\|m u_{i}^{q}+f\right\|_{L^{s}\left(Q_{T}\right)} \\
& \leq C_{0} \bar{m}\left|Q_{T}\right|^{\frac{1}{s}}\left\|u_{i}\right\|_{L^{\infty}\left(Q_{T}\right)}^{q}+C_{0}\|f\|_{L^{s}\left(Q_{T}\right)} \\
& \leq C_{0} \bar{m}\left|Q_{T}\right|^{\frac{1}{s}}\left(2 C_{0}\|f\|_{L^{s}\left(Q_{T}\right)}\right)^{q}+C_{0}\|f\|_{L^{s}\left(Q_{T}\right)} \\
& \leq 2 C_{0}\|f\|_{L^{s}\left(Q_{T}\right)} .
\end{aligned}
$$

By the induction, we see that $\left\|u_{n}\right\|_{L^{\infty}\left(Q_{T}\right)} \leq 2 C_{0}\|f\|_{L^{s}\left(Q_{T}\right)} \leq 2 C_{0} \varepsilon$. Thus, the sequence $\left\{u_{n}\right\}$ is monotone with respect to $n$ and bounded. There exists a function $u_{0}$ such that

$$
\lim _{n \rightarrow \infty} u_{n}(x, t)=u_{0}(x, t), \quad(x, t) \in Q_{T} .
$$


We can verify that $u_{0}$ is a positive periodic solution of the problem (1).

Proof of Theorem 1.1 The non-existence result is shown in Lemma 2.3. We note that

$$
\|f\|_{L^{s}\left(Q_{T}\right)} \leq\left|Q_{T}\right|^{\frac{1}{s}} \sup _{Q_{T}}|f|,
$$

where $\left|Q_{T}\right|$ is the measure of $Q_{T}$. Then the existence result is a simple conclusion of Lemma 2.4.

\section{References}

1. M. J. Esteban, On periodic solutions of superlinear parabolic problems, Trans. Amer. Math. Soc., 293(1986), 171-189.

2. M. J. Esteban, A remark on the existence of positive periodic solutions of superlinear parabolic problems, Proc. Amer. Math. Soc., 102 (1988), 131-136.

3. N. Hirano, N. Mizoguchi, Positive unstable periodic solutions for superlinear parabolic equations, Proc. Amer. Math. Soc., 123(1995), 1487-1495.

4. O. A. Ladyženskaya, V. A. Solonnikov, N. N. Uraltseva, Linear and quasilinear equations of parabolic type, Transl. of Math. Monogr. 23, A.M.S., Providence, R.I., 1968.

5. M. Ôtani, Nonmonotone perturbations for nonlinear parabolic equations associated with subdifferential operators, periodic problems, J. Differential Equations (1984), 248-273.

6. P. Quittner, Multiple equilibria, periodic solutions and a priori bounds for solutions in superlinear parabolic problems, NoDEA Nonlinear Differential Equations Appl., 11(2004), 237-258.

7. Y. F. Wang, J. X. Yin, Z. Q. Wu, Periodic solutions of evolution $p$-Laplacian equations with nonlinear sources, J. Math. Anal. Appl., 219(1998),76-96.

8. J. X. Yin, C. H. Jin, Periodic solutions of the evolutionary $p$-Laplacian with nonlinear sources, J. Math. Anal. Appl., 368(2010), 604-622. 